\title{
Primary Rhabdomyosarcoma of Pancreas: An Extremely Rare Entity
}

\author{
(1) Saida SAKHRI,' (D) Malek BOUHANI,' (D) Olfa JAIDANE,' (D) Nadia SABBEGH ZNAIDI," \\ (D) Riadh CHARGUI, ${ }^{1}$ Khaled RAHAL ${ }^{1}$
}

'Department of Oncologic Surgery, Salah Azaiz Institute, Tunis-Tunus

2Department of Anatomopathology, Charles Nicole Hospital, Tunis-Tunus

\begin{abstract}
SUMMARY
The most recognized soft-tissue sarcoma in children is represented by Rhabdomyosarcoma (RMS). RMS is derived from striated muscle cells or their mesenchymal precursors, with the potential for skeletal muscle differentiation. The most frequent location sites are head and neck, pelvis and extremities. However, the pancreatic location is an unusual circumstance. In this study, we report a case of a 20-yearold woman who was consulted for abdominal pain and severe vomiting. A suspicious pancreatic mass was revealed by radiological investigations. The biopsy with histological exam concluded to RMS of the pancreas. Further investigations showed a metastatic supra-clavicular lymph node. The patient was proposed for biliary stent and palliative chemotherapy, but unfortunately, she passed away five months years following the diagnosis of RMS. However, RMS is frequent in childhood, but the pancreatic location is extremely rare. The physician should keep in mind the primary pancreatic location of rhabdomyosarcoma in young patients.
\end{abstract}

Keywords: Chemotherapy; pancreas; rhabdomyosarcoma.

Copyright $\odot$ 2020, Turkish Society for Radiation Oncology

\section{Introduction}

Rhabdomyosarcoma (RMS) is a malignant tumor of soft tissue raised from the striated muscle cells.[1] RMS occurs more often in children and adolescents [1] and may develop anywhere in the body, even in sites that have no striated muscle tissue. RMS represents $3 \%$ of all the soft tissue sarcomas.[2] Pancreatic location is extremely rare and represents around $0.5 \%$.[3]

\section{Case Report}

In this case study, we report the case of a female aged 20 years who consulted for intense vomiting resistant to treatment and lasting for two months associated with epigastric pain occurring in the context of impaired general condition. Clinical examination showed a lean patient with generalized cutaneous jaundice. Abdominal palpation revealed a $10 \mathrm{~cm}$ epigastric mass with reduced mobility. The examination also showed a left mobile supra-clavicular lymph node measuring $2 \mathrm{~cm}$. Pancreatic enzymes were increased. The abdominopelvic computed tomography (CT) scan revealed a large tissue mass that measured $12 \mathrm{~cm}$ in the pancreatic head encompassing the celiac trunk and especially the superior mesenteric axis with a moderate expansion to the biliary tract and the main bile path (Fig. 1). CT scan also showed many suspect para-aortic lymph nodes. A pancreatic mass biopsy was performed. The histological examination found that the tumor had round cells aspect 


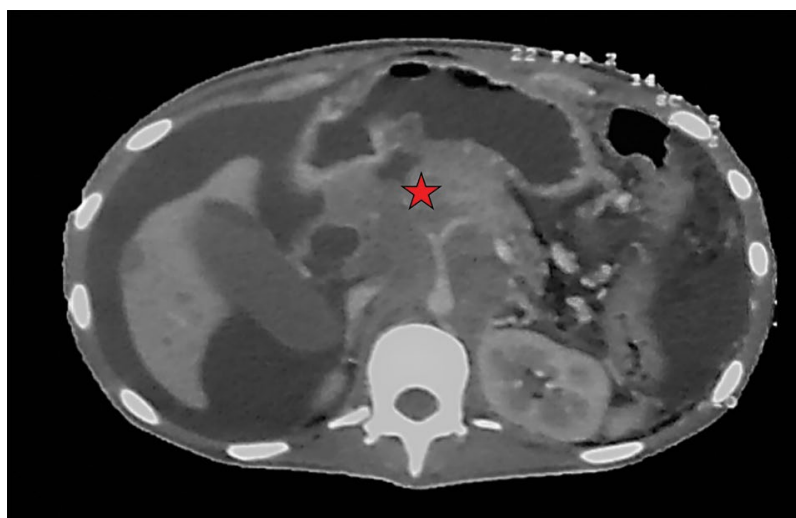

Fig. 1. CT scan showing pancreatic mass (red asterisk).

with an oval nucleus and some mitoses. The immunohistochemical showed tumor positivity for Desmin, Vimentin, and myogenin and negativity for CD117, CD34, S-100, CD68, Melan-A, HMB45, and CK (Fig. 2). The biopsy with histological studies of the supraclavicular lymph node concluded its metastatic nature. Finally, a primary alveolar RMS of the pancreas with lymph node metastasis was confirmed. The decision was to carry out a biliary stent and palliative chemotherapy, but the patient was lost to view. She consulted two months later in an array of impaired general condition with hematemesis requiring transfusion. The evolution was marked by gradual degradation of the general state until death after three months of the discovery of her disease.

\section{Discussion}

RMS was described for the first time by Webner in 1854.[1] RMS develops from the embryonic mes- enchyma with the potential to distinguish into the skeletal muscle.[4] RMS is composed of botryoid, embryonal, alveolar, and pleomorphic subtypes [5] and most commonly arises in the neck, trunk, and extremities, however, primary pancreatic RMS (P-RMS) is a rare entity.[4,6]

P-RMS diagnosis is a real defy for the clinician since the symptoms are unspecific, such as abdominal pain, weight loss, and palpable abdominal mass imitating a choledochal cyst.[1,7] In our case, the mean symptom was intense vomiting with jaundice that leads us to perform directly an abdominopelvic CT scan instead of a gastric endoscopy.

The imaging rarely contributes to the final diagnosis and reveals mostly a non-specific mass with peripheral enhancement. [4] Sometimes, it is difficult to identify P-RMS from other solid pancreatic tumors, which leads to performing a biopsy to establish the diagnosis. $[2,4]$ Pathologic examination reveals large polygonal rhabdomyoblasts. Neoplastic cells show reactivity for Desmin, Myogenin, SMA and vimentin. [3,4] If the tumor is removable, only surgical resection is the curable treatment.[4] It consists of complete excision followed by chemotherapy and radiotherapy.[4] Chemosensitivity is approximately $75 \%$. $[2,4,6]$ The standard regimen is the combination of Vincristine, actinomycin D and Cyclophosphamide (VAC) for both patients with metastasis or not. $[3,4,8]$ Localized disease patients who achieved a pathological response through neoadjuvant chemotherapy may become disease-free after surgery and radiation therapy.[1,3,9]

Long-term prognosis of the P-RMS reported is poor and survival rates are under $50 \%$ in the 1 -year following treatment in adults.[10] We predict a poor prognosis for
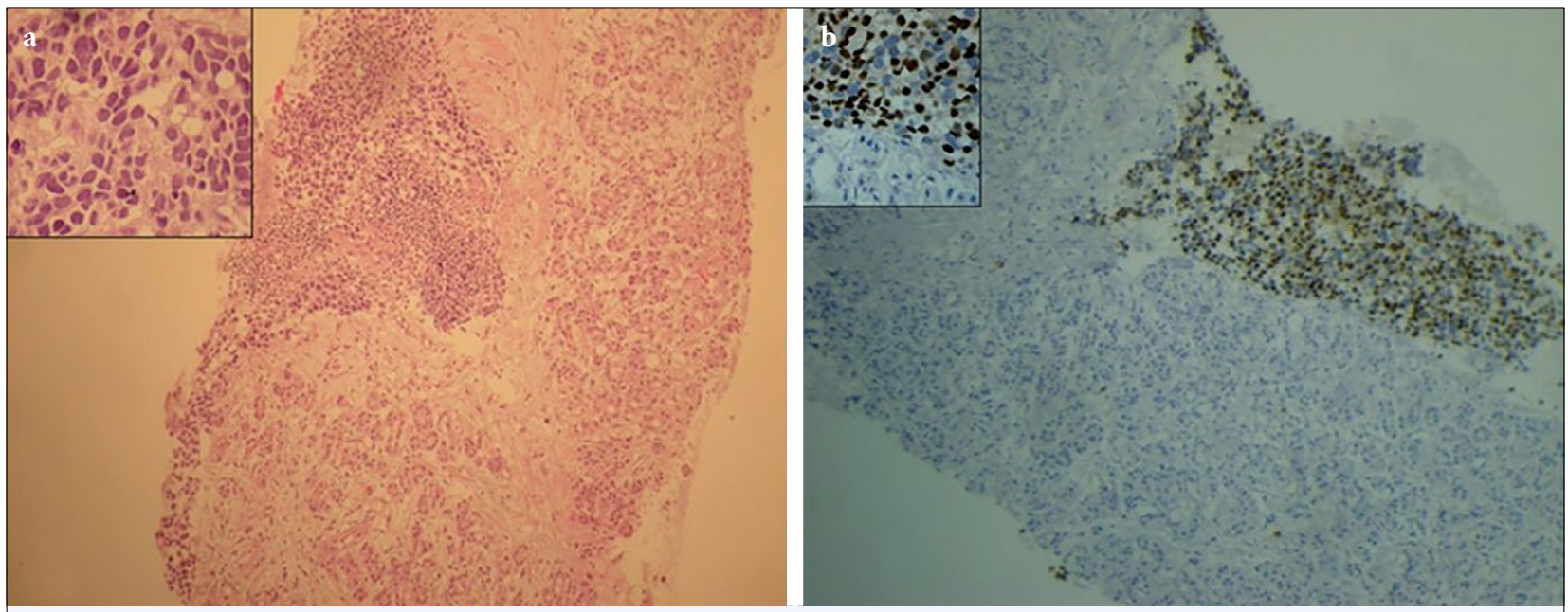

Fig. 2. (a), Hematoxylin and eosin stain showing diffuse tumor proliferation, made of atypical round cells, infiltrating pancreatic acini. (b) Intense and diffuse nuclear expression of myogenin. 
our patient. In metastatic RMS, risk factors by multivariate analysis for very poor survival include old age $(<10$ years), bone or bone marrow involvement, an unfavorable primary site and three or more metastatic sites.[11] Our patient had two risk factors, and according to Oberlin, the 3 -years event-free survival is $18 \%$.[11]

\section{Conclusion}

P-RMS is an extremely rare tumor in adults. The clinician should think of P-RMS as a differential diagnosis for patients with an atypical pancreatic mass. Due to its poor prognosis, more studies are required to establish an optimal therapy.

Informed consent: Written informed consent was obtained from the patient for the publication of the case report and the accompanying images.

Peer-review: Externally peer-reviewed.

Conflict of Interest: No conflict of interest.

Financial Disclosure: There is no financial support from any foundation.

Authorship contributions: Concept - R.C.; Design - M.B.; Supervision - K.R; Materials - N.S.Z.; Data collection \&/or processing - S.S.; Analysis and/or interpretation - O.J.; Literature search - O.J.; Writing - S.S.; Critical review - M.B.

\section{References}

1. Liang W, Xu S. Adult Pancreatic Rhabdomyosarcoma: One Rear Case and Review of the Literature. European Journal of Radiology Extra 2011;79(1):e21-2.

2. Dalfior D, Eccher A, Gobbo S, Brunelli M, Martignoni G, Menestrina F, et al. Primary pleomorphic rhab- domyosarcoma of the kidney in an adult. Ann Diagn Pathol 2008;12(4):301-3.

3. Nemade B, Talapatra K, Shet T, Banavali S, Muckaden MA, Laskar S.Embryohal rhabdomyosarcoma of the biliary tree mimicking a choledochal cyst. J Cancer Res Ther 2007;3(1):40-2.

4. Shirafkan Md A, Boroumand Md N, Komak Md S, Duchini Md A, Cicalese Md L. Pancreatic pleomorphic rhabdomyosarcoma. Int J Surg Case Rep 2015;13:33-6.

5. Pappo AS, Shapiro DN, Crist WM. Rhabdomyosarcoma. Biology and treatment. Pediatr Clin North Am 1997;44(4):953-72.

6. Clark MA, Fisher C, Judson I, Thomas JM. Soft-tissue sarcomas in adults. N Engl J Med 2005;353(7):701-11.

7. Ali S, Russo MA, Margraf L. Biliary rhabdomyosarcoma mimicking choledochal, cyst. J Gastrointestin Liver Dis 2009;18(1):95-7.

8. Jha P, Frölich AM, McCarville B, Navarro OM, Babyn P, Goldsby R, et al. Unusual association of alveolar rhabdomyosarcoma with pancreatic metastasis: emerging role of PET-CT in tumor staging. Pediatr Radiol 2010;40(8):1380-6.

9. Ogilvie CM, Crawford EA, Slotcavage RL, King JJ, Lackman RD, Hartner L, et al. Treatment of adult rhabdomyosarcoma. Am J Clin Oncol 2010;33(2):128-31.

10. Stock N, Chibon F, Binh MB, Terrier P, Michels JJ, Valo I, et al. Adult-type rhabdomyosarcoma: analysis of 57 case with clinicopathologic description, identification of 3 morphologic pattern and prognosis. Am J Surg Pathol 2009;33(12):1850-9.

11. Oberlin O, Rey A, Lyden E, Bisogno G, Stevens MC, Meyer $\mathrm{WH}$, et al. Prognostic factors in metastatic rhabdomyosarcomas: results of a pooled analysis from United States and European cooperative groups. J Clin Oncol 2008;26(14):2384-9. 